SSC18-WKX-05

\title{
The CubeSat Radiometer Radio Frequency Interference Technology Validation (CubeRRT) Mission
}

\author{
Christopher D. Ball, Chi-Chih Chen, Christa J. McKelvey, Graeme E. Smith, Mark Andrews, Andrew J. O’Brien, J. \\ Landon Garry, Joel T. Johnson \\ The Ohio State University \\ 1330 Kinnear Road, Columbus, OH 43212; (614) 292-5752 \\ ball.51@osu.edu \\ Sidharth Misra, Shannon T. Brown, Robert Jarnot, Rudi M. Bendig, Carl Felten \\ NASA Jet Propulsion Laboratory \\ 4800 Oak Grove Drive, Pasadena, CA 91109 \\ sidharth.misra@jpl.nasa.gov \\ Jonathon Kocz \\ California Institute of Technology \\ Pasadena, CA 91125 \\ jkocz@astro.caltech.edu
}

Jeffrey R. Piepmeier, Damon C. Bradley, Priscilla N. Mohammed, Jared F. Lucey, Kevin A. Horgan, Quenton Bonds, Carlos E. Duran-Aviles, Michael A. Solly, Matthew A. Fritts

\author{
NASA Goddard Space Flight Center \\ Greenbelt, MD 20771 \\ jeff.piepmeier@nasa.gov
}

Doug Laczkowski, Matthew Pallas, Ervin Krauss

Blue Canyon Technologies,

5330 Airport Blvd, Ste 100, Boulder, CO 80301

dlaczkowski@bluecanyontech.com

\begin{abstract}
The CubeSat Radiometer Radio Frequency Interference Technology Validation (CubeRRT) mission has developed a $6 \mathrm{U}$ CubeSat to demonstrate radio frequency interference (RFI) detection and mitigation technologies for future Earth remote sensing missions. Anthropogenic sources of RFI can degenerate important geophysical retrievals from spaceborne passive microwave radiometers. Real-time on-board RFI processing is therefore an important technology needed for future radiometry missions. CubeRRT will perform microwave radiometry observations in $1 \mathrm{GHz}$ channels tunable from 6-40 GHz and will demonstrate on-board real-time RFI processing. The CubeRRT payload consists of a wideband antenna subsystem developed at Ohio State, a tunable analog radiometer subsystem developed at Goddard Space Flight Center, and a digital backend processor for real-time RFI mitigation developed at the Jet Propulsion Laboratory. The spacecraft bus was developed and integrated at Blue Canyon Technologies. The enabling CubeRRT technology is a digital Field-Programmable Gate Array-based spectrometer with $1 \mathrm{GHz}$ bandwidth that implements advanced RFI filtering algorithms based on real-time kurtosis and cross-frequency techniques. CubeRRT was manifested on the OA-9 International Space Station resupply mission and launched on May 21, 2018. This talk will describe the assembly and test of the flight system as well as the status of on-orbit operations.
\end{abstract}

\section{INTRODUCTION}

In recent years, satellite-based passive microwave radiometry measurements have exhibited increasing levels of anthropogenic interference. ${ }^{1,2}$ This radio frequency interference (RFI) can corrupt geophysical retrievals for a variety of crucial science products, including soil moisture, atmospheric water vapor, sea surface temperature, sea surface winds, and many others Spectrum for commercial use is becoming increasingly crowded, accelerating demand to open the bands reserved for passive microwave Earth observation and radio astronomy applications to general use. Due to current shared spectrum allocations, microwave radiometers must co-exist with terrestrial RFI sources, 
often resulting in RFI corruption as demonstrated in numerous past missions. As active sources expand over larger areas and occupy additional spectrum, microwave radiometry becomes increasingly difficult without an RFI mitigation capability. Co-existence in some cases should be possible if such RFI mitigation technology is included in future satellite-based systems.

Initial progress in RFI detection and filtering technologies for microwave radiometry in space has been achieved in the SMAP mission. ${ }^{3-5}$ The SMAP radiometer utilizes a digital subsystem that operates on a $24 \mathrm{MHz}$ bandwidth, centered at the protected $1413 \mathrm{MHz}$ frequency allocation. However, RFI mitigation for microwave radiometry at higher frequencies (e.g., 6-40 $\mathrm{GHz}$ ) requires greater bandwidth, increasing the overall processing power, instantaneous bandwidth, and power consumption of the digital subsystem. While the SMAP mission is demonstrating RFI filtering in a single 24 $\mathrm{MHz}$ channel, all RFI processing is performed on the ground following downlink of high data rate products such as a spectrogram of the received signal and its kurtosis. The multiple channels and much larger bandwidths of current and future radiometer missions operating over 6-40 GHz do not allow downlink of this data volume to occur, so that RFI processing on the ground is not possible. Real-time, on-board RFI processing is therefore an important technology needed for future missions.

To demonstrate on-board, real-time RFI processing from 6-40 GHz, the CubeRRT mission was selected under NASA's In-space Validation of Earth Science Technologies (InVEST) program. ${ }^{6}$ This paper focuses on the development of the CubeRRT spacecraft and payload instrument as well as discussion of on-orbit operations. The CubeRRT satellite was delivered in March 2018 in preparation for launch on the OA-9 resupply mission to the International Space Station (ISS). The OA-9 mission was launched successfully in May 2018.

\section{MISSION OBJECTIVES}

The CubeRRT radiometer is designed to make wideband measurements in ten distinct bands relevant to passive microwave remote sensing in the range of $6 \mathrm{GHz}$ to 40 GHz. The RFI mitigation technology was demonstrated on the ground using flight-ready hardware, thereby advancing the Technology Readiness Level (TRL) from 5 to 6. Successful demonstration of the technology in space will further elevate TRL from 6 to 7 . The CubeRRT mission intends to quantify the capability of the wideband digital RFI filtering technology to detect and remove pulsed and continuous sinusoidal RFI with a power level of 2 times the noise equivalent delta temperature (NEDT) or greater for a Nyquist bandwidth of $1 \mathrm{GHz}$. The baseline CubeRRT mission will provide radiometry data from at least 100 hours of spaceborne operation, including at least 10 hours of spaceborne operation in each of ten common radiometry bands.

\section{SYSTEM HARDWARE}

The CubeRRT satellite system is composed of a spacecraft bus, which provides the power, command, communications, and control elements of the system, and the payload instrument, which houses the CubeRRT radiometer and RFI processor.

\section{CubeRRT spacecraft}

The CubeRRT 6U spacecraft bus was designed, constructed, and tested by Blue Canyon Technologies (BCT). Figure 1 shows a concept drawing of CubeRRT in its deployed configuration. Key features of the spacecraft include the bus power system (solar panels, batteries), an isolated instrument power supply to mitigate ground loops in the radiometer payload, data communications systems (Cadet radio and Globalstar satellite communications), the mechanical frame, and additional avionics components for attitude control and other functions.

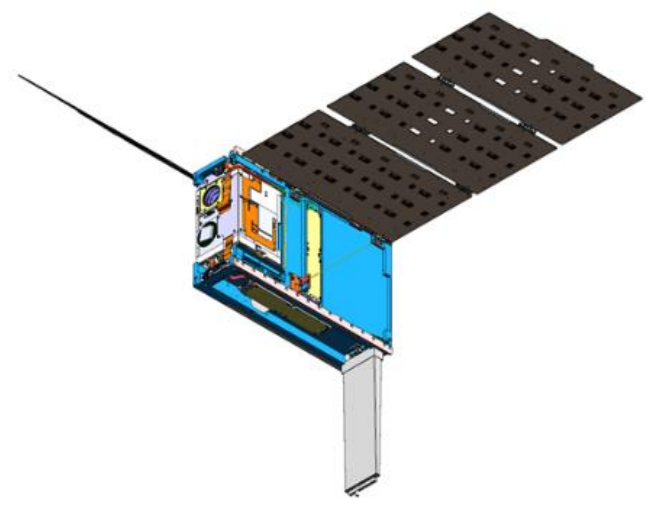

Figure 1. Illustration of the CubeRRT 6U spacecraft in orbit deployed configuration

\section{Payload instrument}

The CubeRRT payload consists of 3 subsystems which work together to provide the radiometry measurements used to execute the CubeRRT mission. ${ }^{7}$ The payload antenna subsystem is composed of three wideband tapered helical antennas developed by The Ohio State University (OSU) to cover the frequency range of 6-40 $\mathrm{GHz}$. A tunable analog radiometer front-end (RFE) subsystem was developed by NASA Goddard Space Flight Center (GSFC). The RFE is tunable from 6 to 40 $\mathrm{GHz}$ with a $1 \mathrm{GHz}$ bandwidth centered at an IF frequency of $1.5 \mathrm{GHz}$. Lastly, the radiometer's digital backend (RDB) was developed by NASA Jet Propulsion Laboratory (JPL). The RBD reports a 128-point 
spectrum of its $1 \mathrm{GHz}$ bandwidth, performs real-time RFI detection, and reports unmitigated and RFImitigated total power. Figure 2 shows the RFE and RDB subsystems mounted to a baseplate for integrated payload testing.

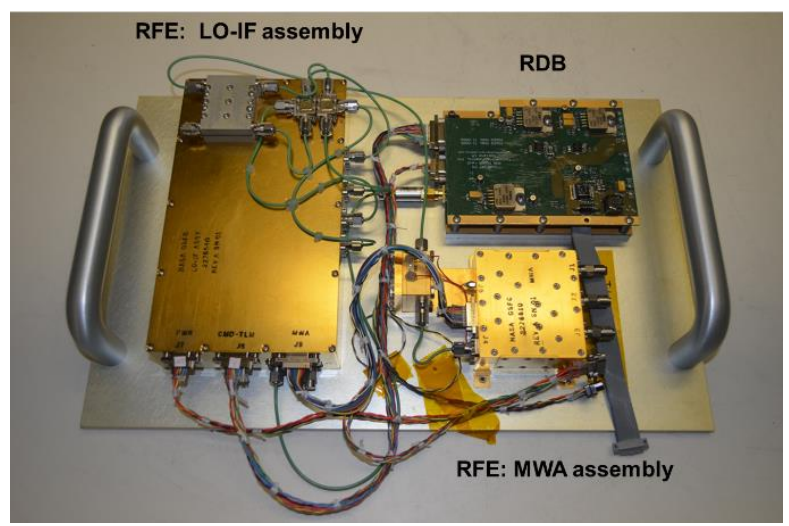

Figure 2. CubeRRT RFE and RDB subsystems, integrated for payload testing

\section{SYSTEM TESTING}

The CubeRRT instrument has undergone extensive testing to ensure its capability for meeting mission requirements. Flight model testing payload occurred in October 2017. The four objectives of this test effort were to make end-to-end radiometry measurements through the payload, to implement and optimize passband flattening, to demonstrate successful RFI detection and mitigation using injected and radiated signals, and to execute the thermal cycle test profile.

The results of an injected signal demonstration are shown in Figures 3-5. In this example, the radiometer was tuned to $10.4 \mathrm{GHz}$ and a low-power $\mathrm{CW}$ sinusoidal signal of constant amplitude was injected into the antenna port of the RFE. This synthetic RFI signal was stepped across the instrument's passband in $100 \mathrm{MHz}$ steps. Figure 3 was generated by normalizing the second moment of each of the 128 frequency bins to their respective means. The injected RFI is visible in the plot as short, bright lines at fixed frequencies with a length of approximately 400 packets. Figure 4 shows the RFI flags generated by the RDB using kurtosis and crossfrequency detection algorithms. These flags were determined by manual inspection to match the injected RFI frequencies and times.

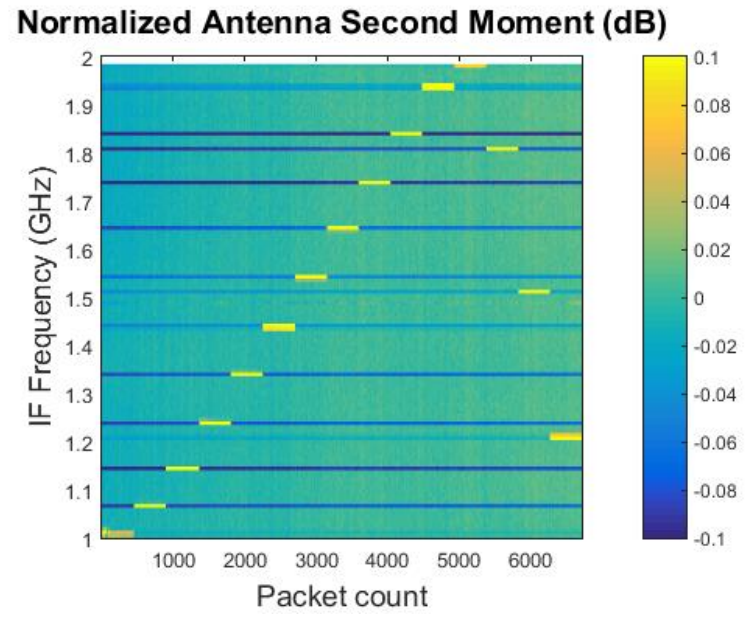

Figure 3. Normalized second moment with RFI injected and stepped in frequency

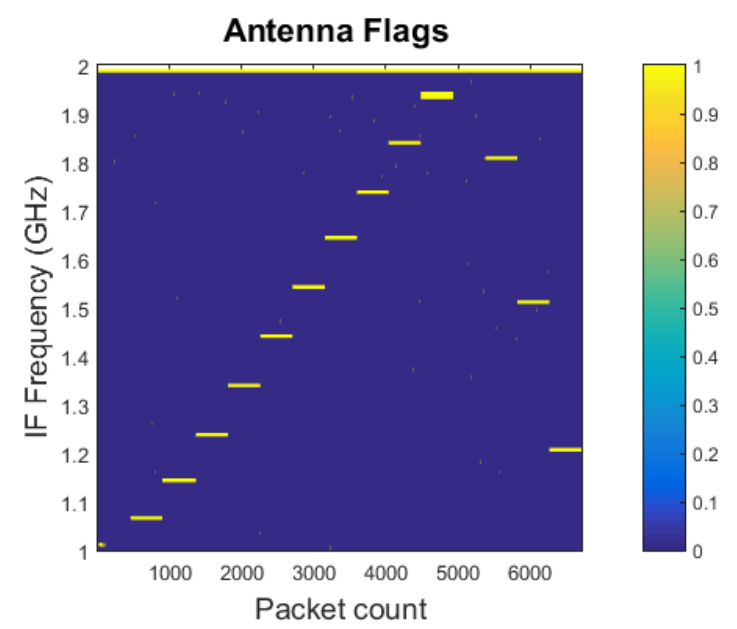

Figure 4. RFI flags reported by RDB

The RDB uses the flags to remove RFI-corrupted data from its aggregated output. Figure 5 shows the resulting brightness temperatures, with and without RFI mitigation, revealing that the injected RFI contributed excess brightness temperatures ranging from $\sim 2-10 \mathrm{~K}$ in this experiment (depending on the source location within the instrument passband), and that the excess signal was successfully mitigated by the RFI processor. 


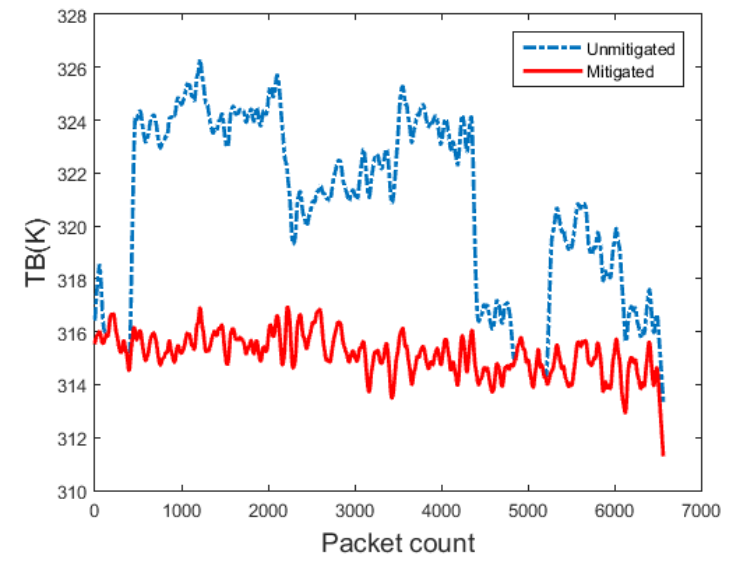

Figure 5. Brightness temperature, with RFI ("unmitigated") and with RFI removed ("mitigated").

\section{CONCEPT OF OPERATION}

CubeRRT's mission goals of evaluating on-board detection and filtering of RFI, coupled with the limited solar cell and battery capacity of a 6U CubeSat, bring about unique challenges for payload operations. These challenges primarily reside with regard to scheduling the power cycling and frequency tuning of the payload. In contrast to other radiometer missions, which typically aim to gather brightness temperature information of the entire Earth's surface, CubeRRT's power budget allows operation only at a duty cycle of approximately $30 \%$. The system prioritizes operation of the payload at locations of known RFI sources and over landmasses where RFI is more likely to occur.

To assist in the modelling of these operations, OSU developed a scheduler simulation tool. This tool is used to develop algorithms for power cycling and frequency tuning, radiometry coverage maps, battery depth-ofdischarge (DOD) and payload data or telemetry profiles. In addition, the scheduler automates the process of generating payload command sequences for regular uplinking to the spacecraft.

To properly model CubeRRT operations, the scheduler simulates the power system, telemetry buffers, RFI coordinates, and orbital propagation models. The power system state is modelled with knowledge of the available energy from CubeRRT's solar cells, the known power draw from the satellite bus and payload subsystems, and the battery capacity. Telemetry and payload data buffers are monitored and downlinked at a known rate to a ground station at Wallops Flight Facility (WFF) to predict and prevent buffer overflow conditions. The current set of RFI locations is generated from existing radiometry datasets produced by previous nadirobserving missions (Jason and TRMM).
The general algorithm for power cycling divides payload operation into three 10 minute blocks within a $\sim 90$ minute orbit, resulting in a $30 \%$ duty cycle as illustrated in Figure 6. The payload orients the three blocks by first prioritizing observation at known RFI locations, followed by land observation. If RFI and land are exhausted for a particular orbit, operation during maximum solar availability is then selected. If the chosen sequence exceeds a battery depth of discharge threshold, the power sequence reverts to operating in peak sun. While the payload is operating, the payload sequentially sweeps through the ten radiometer bands in $\sim 15$-second increments.

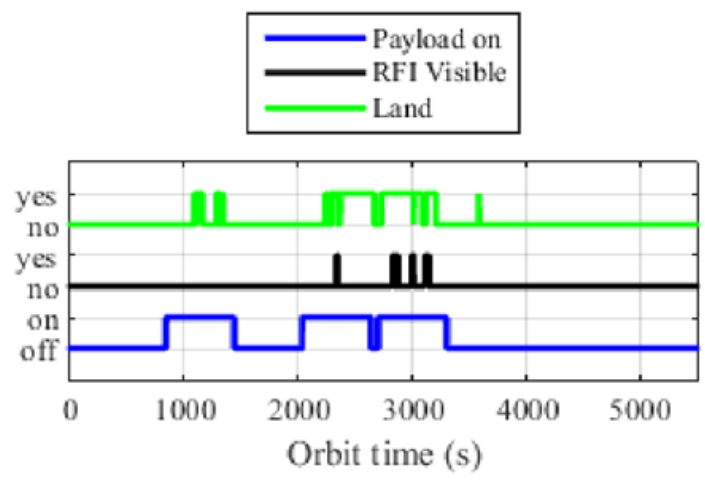

Figure 6. Illustration of orbit power cycling

This scheduling algorithm was propagated over the one year expected mission lifetime, with results shown in Figure 7. The payload exhibits an RFI efficiency of approximately $98 \%$, defined as the percentage of time the payload operates when known RFI points are visible within the radiometer's footprint. The payload will spend approximately $65 \%$ of its operational time observing landmasses. It will observe approximately 99\% of available land (excluding the South Atlantic Anomaly around eastern South America) within the latitude limits of an ISS deployed orbit at least once over the year of operation.

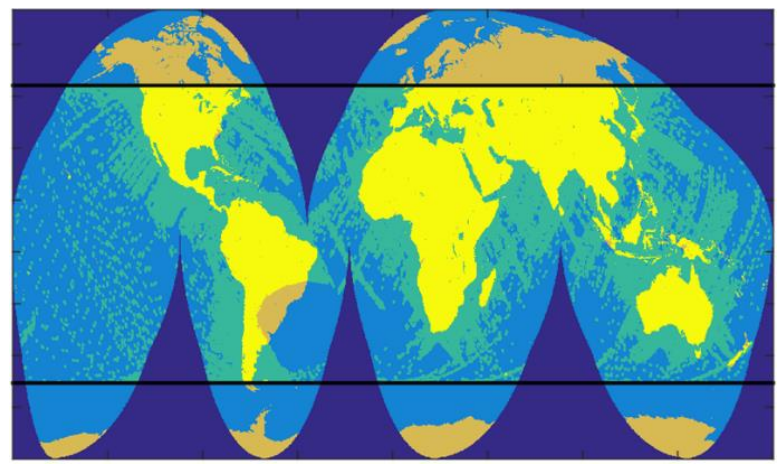

Figure 7. CubeRRT coverage at $6.8 \mathrm{GHz}$ following one year of operations 
The general workflow for uplinking commands and downlinking telemetry and payload data is shown in Figure 8. OSU will generate daily Planning Action Lists (PALs) based on running the CubeRRT scheduler. The PALs are sent to BCT to develop and validate the spacecraft command list, which is then sent to WFF for uplink to CubeRRT on the next available pass. Downlinked data is sent from WFF to BCT, where it is parsed and archived accordingly. OSU then processes the payload data and telemetry to verify that the radiometer is functioning nominally and that the backend processor is detecting and filtering RFI accurately. The final data product will include frequencies, unmitigated and mitigated brightness temperatures, latitude, longitude, pixel size, time, and RFI flags. These data products will be publicly available via web access to an OSU-hosted site (https://u.osu.edu/cuberrt/).

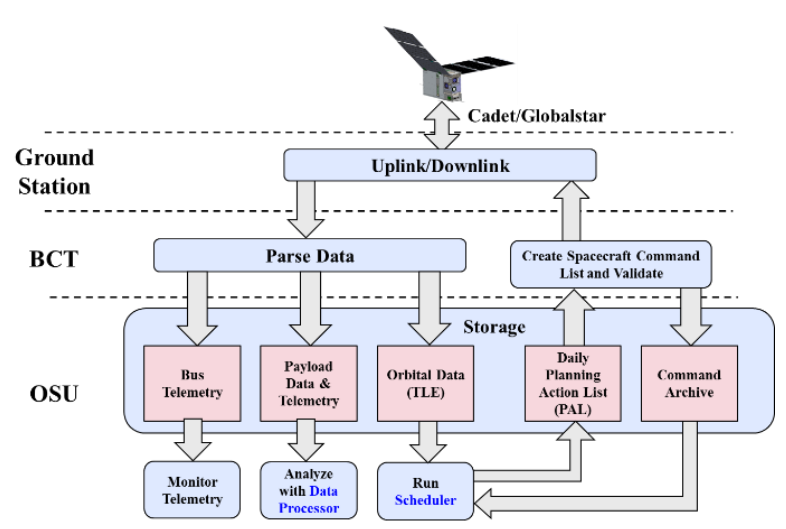

Figure 8. Process flow of CubeRRT operations, including commanding and data retrieval

\section{CONCLUSIONS AND NEXT STEPS}

The CubeRRT system was designed to meet an ambitious set of mission objectives in order to demonstrate and mature spaceborne RFI mitigation technology. CubeRRT was launched in May 2018 on the OA-9 mission, docking successfully with ISS. Following deployment of CubeRRT into orbit, a commissioning phase will commence in which all systems are verified in advance of normal operations. Payload commanding will be developed by the OSU team, passed along to BCT for processing, and uplinked to CubeRRT via the WFF ground station. Downlinked telemetry and payload data will be received by WFF, processed initially by BCT, and handed off to OSU for ground-based verification of the RFI mitigation capability. A total of one year of mission operations is anticipated.

\section{References}

1. Spectrum Management for Science in the 21st Century, M. H. Cohen and A. J. Gasiewski (cochairs), National Research Council, 2010.
2. Presidential Memorandum: Unleashing the Wireless Broadband Revolution, June 28th, 2010. (http://www.whitehouse.gov/the-pressoffice/presidential-memorandum-unleashingwireless-broadband-revolution).

3. D. Entekhabi et al., "The Soil Moisture Active Passive (SMAP) Mission," in Proceedings of the IEEE, vol. 98, no. 5, pp. 704-716, May 2010.

4. J. R. Piepmeier et al., "Radio-Frequency Interference Mitigation for the Soil Moisture Active Passive Microwave Radiometer," in IEEE Transactions on Geoscience and Remote Sensing, vol. 52, no. 1, pp. 761-775, Jan. 2014.

5. P. N. Mohammed, J. R. Piepmeier, J. T. Johnson, and O. Salama, "Performance of the RFI detection and mitigation algorithms for the soil moisture active/passive (SMAP) radiometer," IGARSS, 2014.

6. J. T. Johnson et al., "The CubeSat Radiometer Radio Frequency Interference Technology Validation (CubeRRT) mission," 2016 IEEE International Geoscience and Remote Sensing Symposium (IGARSS), Beijing, 2016, pp. 299-301.

7. C. Ball et al., "Development of the CubeSat radiometer radio frequency interference technology validation (CubeRRT) system," 2017 IEEE International Geoscience and Remote Sensing Symposium (IGARSS), Fort Worth, TX, 2017, pp. 5942-5945. 